\title{
Kinetic decoupling of neutralino dark matter
}

\author{
Xuelei Chen* \\ Department of Physics, The Ohio State University, 174 West 18th Avenue, Columbus, Ohio 43210 \\ Marc Kamionkowski \\ California Institute of Technology, Mail Code 130-33, Pasadena, California 91225 \\ Xinmin Zhang \\ Institute of High Energy Physics, Chinese Academy of Sciences, P.O. Box 918-4, Beijing 100039, People's Republic of China
}

(Received 27 March 2001; published 25 June 2001)

\begin{abstract}
After neutralinos cease annihilating in the early Universe, they may still scatter elastically from other particles in the primordial plasma. At some point in time, however, they will eventually stop scattering. We calculate the cross sections for neutralino elastic scattering from standard-model particles to determine the time at which this kinetic decoupling occurs. We show that kinetic decoupling occurs above a temperature $T$ $\sim \mathrm{MeV}$. Thereafter, neutralinos act as collisionless cold dark matter.
\end{abstract}

DOI: 10.1103/PhysRevD.64.021302 PACS number(s): 98.80.Cq, 13.15. $+\mathrm{g}, 12.60 . J v, 95.35 .+\mathrm{d}$

\section{INTRODUCTION}

Neutralinos provide perhaps the most promising candidate for the mysterious dark matter in galactic halos (see, e.g., Refs. [1,2] for reviews). Such particles would have existed in thermal equilibrium in the early Universe when the temperature exceeded the mass of the particle. However, as the temperature drops below the mass, the rate of the production and destruction reactions that maintain the equilibrium density become smaller than the expansion rate. At this point, annihilations cease, and a relic population of neutralinos remains. The calculation of this relic density is straightforward, and to a first approximation, the relic density is fixed by the cross section for neutralino annihilation to lighter standard-model particles. The cross section required for the critical density is $\sim 10^{-37} \mathrm{~cm}^{2}$, coincidentally within a few orders of magnitude of the neutralino annihilation cross section in the minimal supersymmetric extension of the standard model.

After freezeout (i.e., after they fall out of chemical equilibrium) neutralinos may still continue scattering elastically from other particles in the primordial plasma, and thus remain in chemical equilibrium. In an interesting recent paper [3], Bœhm et al. pointed out that if these particles remain in kinetic equilibrium until a temperature $T \sim 0.1 \mathrm{keV}$, at which point the mass enclosed in a Hubble volume is comparable to that of a dwarf galaxy, then collisional damping due to elastic scattering of neutrinos and/or photons from neutralinos could erase structure on such scales and thus help resolve the discrepancy between the predicted and observed substructure in Galactic halos [4,5]. They also argued briefly that supersymmetric dark-matter candidates may have the required neutralino-photon and/or neutralino-neutrino cross sections.

When heavy neutrinos were first considered as cold dark

\footnotetext{
*Electronic address: xuelei@pacific.mps.ohio-state.edu

†Electronic address: kamion@tapir.caltech.edu

\#Electronic address: xmzhang@alpha02.ihep.ac.cn
}

matter candidates, Gunn et al. [6] showed that they would fall out of kinetic equilibrium shortly after their annihilation freezes out. Later, Schmid et al. [7] estimated the decoupling temperature of neutralino-neutrino interaction. In this paper we consider the process of kinetic decoupling of neutralinos in more detail. We calculate the cross sections for elastic scattering of neutrinos and photons from neutralinos, and for completeness, we consider the elastic scattering of neutralinos from other particles as well. We show that the estimates for neutrino-neutralino and photon-neutralino scattering used by Bœhm et al. neglect important kinematic effects, and thus hugely overestimate these cross sections. We show that neutralinos are more likely to undergo kinetic freezeout much earlier, at temperatures between a $\mathrm{MeV}$ and a $\mathrm{GeV}$, rather than a keV. Thus, although Bœhm et al.'s mechanism for collisional damping may be applicable to more general darkmatter candidates, it is unlikely to be relevant to the most promising supersymmetric dark-matter candidates.

In the next section we briefly review the cross sections required for the Bœhm et al. collisional damping. In Sec. III, we calculate the cross section for neutralino-photon scattering. In Sec. IV, we move on to the neutralino-neutrino cross section. We consider elastic scattering of neutralinos from other species in Sec. V, and we conclude in Sec. VI.

\section{REQUIRED CROSS SECTIONS}

In the standard scenario, neutrinos undergo chemical decoupling at a temperature of one $\mathrm{MeV}$ and their kinetic decoupling follows shortly thereafter. Now suppose that the neutrino has a nonzero elastic-scattering cross section with the dark-matter particle $\chi$. This elastic scattering will keep the dark-matter particles in kinetic equilibrium until the rate at which neutrinos scatter from a given dark-matter particle becomes comparable to the expansion rate. The scattering rate is $\Gamma=n_{\nu} \sigma_{\nu \chi} c$, where $n_{\nu}$ is the number density of neutrinos and $\sigma_{\nu \chi}$ is the neutrino-dark-matter elastic-scattering cross section. The Hubble expansion rate is $H$ $=1.66 g_{*}^{1 / 2} T^{2} / m_{\mathrm{Pl}}$, where $g_{*}=3.36$ is the effective number 


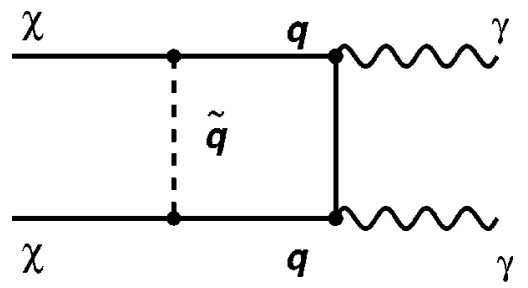

FIG. 1. One of the Feynman diagrams for neutralino annihilation to two photons. There are also other Feynman diagrams for this process.

of relativistic degrees of freedom at a temperature $T \sim \mathrm{keV}$, and $m_{\mathrm{Pl}} \simeq 10^{19} \mathrm{GeV}$ is the Planck mass. The temperature at which the Hubble volume encloses a baryonic mass $M_{b}$ is

$$
T=0.15 \operatorname{keV}\left(M_{b} / 10^{9} M_{\odot}\right)^{-1 / 3}\left(\Omega_{b} h^{2} / 0.02\right)^{1 / 3},
$$

where $\Omega_{b}$ is the baryon density and $h$ is the present Hubble parameter in units of $100 \mathrm{~km} \mathrm{sec}^{-1} \mathrm{Mpc}^{-1}$. Setting $\Gamma=H$, at $T \sim 0.1 \mathrm{keV}$, we find that the cross section required to erase structure on $\lesssim 10^{9} M_{\odot}$ scales is $\sigma_{\nu \chi} \sim 10^{-40} \mathrm{~cm}^{2}$, which recovers the estimate of Bœhm et al. Similar arguments for scattering from photons lead Bœhm et al. to estimate a dark-matter-photon cross section of $10^{-38} \mathrm{~cm}^{2}$ for collisional damping via photon scattering.

In fact, we believe that Bœhm et al. may have underestimated the required cross section by several orders of magnitude. The cold-dark-matter particles are non-relativistic, so even if neutrino and photon scattering keep them in kinetic equilibrium, they could hardly move far enough to smooth out fluctuations in the dark-matter density. For collisional damping to work, the motion of photons and neutrinos must be affected. Indeed, this is how collisional damping works in the case of baryons (Silk damping) in which density perturbations are smoothed on scales which are small compared with the photon diffusion length [8]. If it is in fact the neutrinos (or photons) that are responsible for collisional damping with neutralino-neutrino scattering, then the appropriate reaction rate to equate to the Hubble length is the rate at which a given neutrino scatters from a neutralino, rather than vice versa. In this case, the required neutrino-dark-matter cross section must be larger by the ratio of the neutrino number density to the dark-matter number density, and this factor is $5 \times 10^{7}\left(m_{\chi} / \mathrm{GeV}\right)$, where $m_{\chi}$ is the dark-matter-particle mass.

\section{NEUTRALINO-PHOTON SCATTERING}

The cross section for annihilation of two neutralinos to two photons has been calculated by a number of authors (e.g., Refs. [9-14]). Annihilation can proceed, e.g., through a Feynman diagram such as that shown in Fig. 1, but there are also many other diagrams that can contribute. The value of the annihilation cross section depends on a number of supersymmetric couplings and sparticle masses, and it may span a range of several orders of magnitude. In some regions of the plausible supersymmetric parameter space, it can indeed be as large as $10^{-38} \mathrm{~cm}^{2}$ [13].

The diagrams for elastic scattering of photons from neu- tralinos are related to these by crossing symmetry, and this led Bœhm et al. to speculate that the elastic-scattering cross sections could also be as large as $10^{-38} \mathrm{~cm}^{2}$. This, however, neglects a dramatic suppression of the elastic-scattering cross section that arises from the spinor structure of the amplitude.

The amplitude for either process can be written,

$$
\mathcal{A}=\frac{e^{2}}{4 \pi^{2}} \varepsilon_{\mu \nu \rho \sigma} k^{\mu} k^{\nu} \epsilon^{\rho} \epsilon^{\sigma} \overline{\mathcal{A}},
$$

where $k^{\mu}$ and $k^{\nu}$ are the four-momenta of the photons and $\epsilon^{\rho}$ and $\epsilon^{\sigma}$ are their polarization four-vectors, and $\varepsilon_{\mu \nu \rho \sigma}$ is the completely antisymmetric tensor. The reduced amplitude $\overline{\mathcal{A}}$ depends on the particle masses and couplings in the diagram loops. Its value for annihilation will differ from that for elastic scattering by factors of order unity, but not by many orders of magnitude. For the annihilation process, the fourmomenta will be equal to $m_{\chi}$, while for elastic scattering, they will be equal to the photon energy. Thus, neglecting the small difference in $\overline{\mathcal{A}}$, we see that the factors of $k$ in the amplitude will lead to a reduction by a factor $\left(E_{\gamma} / m_{\chi}\right)^{4}$ of the elastic-scattering cross section relative to that for annihilation. For $E_{\gamma} \sim 0.1 \mathrm{keV}$ and a neutralino mass $m_{\chi}$ $\sim 100 \mathrm{GeV}$, this suppression is $10^{-36}$. More precisely, the annihilation cross section is

$$
\sigma_{\chi \chi \rightarrow \gamma \gamma}=\frac{\alpha^{2} m_{\chi}^{2}}{16 \pi^{3}}|\overline{\mathcal{A}}|^{2},
$$

while the elastic-scattering cross section is

$$
\sigma_{\chi \gamma \rightarrow \chi \gamma}=\frac{\alpha^{2}}{3 \pi^{2} m_{\chi}^{2}} E_{\gamma}^{4}|\overline{\mathcal{A}}|^{2}
$$

We have calculated this cross section for a number of supersymmetric models using a modified version of the code NEUTDRIVER [1]. For a range of typical supersymmetric parameters and a photon energy of $100 \mathrm{eV}$, we find $10^{-79} \mathrm{~cm}^{2}<\sigma<10^{-73} \mathrm{~cm}^{2}$. We thus conclude that elastic neutralino-photon scattering will play no role in maintaining kinetic equilibrium of neutralinos, nor of scattering photons from neutralinos.

\section{NEUTRALINO-NEUTRINO SCATTERING}

The neutrino-neutralino differential scattering cross section is given by

$$
\left(\frac{d \sigma}{d \Omega}\right)=\frac{|\mathcal{M}|^{2}}{64 \pi^{2} m_{\chi}^{2}},
$$

where

$$
\mathcal{M}=\mathcal{M}_{s}+\mathcal{M}_{t}+\mathcal{M}_{u}
$$

is the scattering amplitude. The Feynman diagrams are shown in Fig. 2 (we have omitted Higgs-boson-exchange 


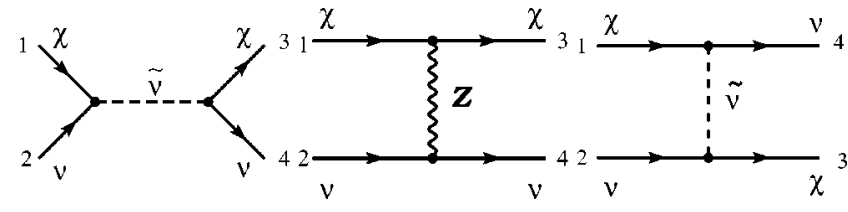

FIG. 2. Feynman diagrams for elastic neutralino-neutrino scattering.

diagrams since the Yukawa coupling of the Higgs boson is zero in the limit of zero neutrino mass). These are given by ${ }^{1}$

$$
\begin{gathered}
\mathcal{M}_{s}=i \frac{\left|X_{\nu i j 0}^{\prime}\right|^{2}}{s-m_{\tilde{\nu}_{j}}^{2}} \bar{u}_{4} P_{R} C \bar{u}_{3}^{T} u_{1}^{T} C^{-1} P_{L} u_{2}, \\
\mathcal{M}_{t}=i \frac{g^{2}}{2 \cos ^{2} \theta_{w}} \frac{1}{t-m_{Z}^{2}} \bar{u}_{4} \gamma^{\mu} P_{L} u_{2} \bar{u}_{3} \gamma_{\mu}\left(O_{00 L}^{\prime \prime} P_{L}\right. \\
\left.+O_{00 R}^{\prime \prime} P_{R}\right) u_{1}, \\
\mathcal{M}_{u}=-i \frac{\left|X_{\nu i j 0}^{\prime}\right|^{2}}{u-m_{\tilde{\nu}_{j}}^{2}} \bar{u}_{4} P_{R} u_{1} \bar{u}_{3} P_{L} u_{2},
\end{gathered}
$$

where $i, j$ are the flavor indices of the neutrino and sneutrino, respectively, $P_{L}=\left(1-\gamma_{5}\right) / 2$ and $P_{R}=\left(1+\gamma_{5}\right) / 2, X_{\nu i j 0}^{\prime}$ is the neutralino-sneutrino-neutrino coupling (given in Ref. [1]), and $O_{00 R}^{\prime \prime}$ and $O_{00 L}^{\prime \prime}$ are neutralino- $Z^{0}$ couplings (also given in Ref. [1]). The superscript $T$ denotes the transpose of the matrix, and $C$ is the charge-conjugation matrix. Then,

$$
\begin{aligned}
|\mathcal{M}|^{2}= & \left|\mathcal{M}_{s}\right|^{2}+\left|\mathcal{M}_{t}\right|^{2}+\left|\mathcal{M}_{u}\right|^{2}+2 \Re\left(\mathcal{M}_{s} \mathcal{M}_{t}^{*}+\mathcal{M}_{s} \mathcal{M}_{u}^{*}\right. \\
& \left.+\mathcal{M}_{t} \mathcal{M}_{u}^{*}\right)
\end{aligned}
$$

The various terms are given

$$
\begin{aligned}
\left|\mathcal{M}_{s}\right|^{2}= & \frac{2\left|X_{\nu i j 0}^{\prime}\right|^{4}}{\left(s-m_{\tilde{\nu}_{j}}^{2}\right)^{2}}\left(p_{1} p_{2}\right)\left(p_{3} p_{4}\right), \\
\left|\mathcal{M}_{t}\right|^{2}= & \frac{2 g^{4}}{\cos ^{4} \theta_{w}\left(t-m_{Z}^{2}\right)^{2}}\left[\left|O_{00 L}^{\prime \prime}\right|^{2}\left(p_{1} p_{2}\right)\left(p_{3} p_{4}\right)\right. \\
& \left.+\left|O_{00 R}^{\prime \prime}\right|^{2}\left(p_{1} p_{4}\right)\left(p_{2} p_{3}\right)-O_{00 L}^{\prime \prime} O_{00 R}^{\prime \prime} m_{\chi}^{2}\left(p_{2} p_{4}\right)\right],
\end{aligned}
$$

$$
\left|\mathcal{M}_{u}\right|^{2}=\frac{2\left|X_{\nu i j 0}^{\prime}\right|^{4}}{\left(u-m_{\tilde{\nu}_{j}}^{2}\right)^{2}}\left(p_{1} p_{4}\right)\left(p_{2} p_{3}\right),
$$

\footnotetext{
${ }^{1}$ For Feynman rules of Majorana fermions, see, e.g., Ref. [15].
}

$$
\begin{aligned}
\mathcal{M}_{s} \mathcal{M}_{t}^{*}= & -\frac{g^{2}}{\cos ^{2} \theta_{w}} \frac{\left|X_{\nu i j 0}^{\prime}\right|^{2}}{s-m_{\tilde{\nu}_{j}}^{2}} \frac{1}{t-m_{\tilde{\nu}_{j}}^{2}}\left[2 O_{00 L}^{\prime \prime *}\left(p_{1} p_{2}\right)\left(p_{3} p_{4}\right)\right. \\
& \left.-O_{00 R}^{\prime \prime *} m_{\chi}^{2}\left(p_{2} p_{4}\right)\right] \\
\mathcal{M}_{s} \mathcal{M}_{u}^{*}= & -\frac{\left|X_{\nu i j 0}^{\prime}\right|^{4}}{\left(s-m_{\tilde{\nu}_{j}}^{2}\right)\left(u-m_{\tilde{\nu}_{j}}^{2}\right)} m_{\chi}^{2}\left(p_{2} p_{4}\right) \\
\mathcal{M}_{t} \mathcal{M}_{u}^{*}= & -\frac{g^{2}}{\cos ^{2} \theta_{w}} \frac{\left|X_{\nu i j 0}^{\prime}\right|^{2}}{\left(t-m_{Z}^{2}\right)\left(u-m_{\tilde{\nu}_{j}}^{2}\right)}\left[2 O_{00 R}^{\prime \prime}\left(p_{1} p_{4}\right)\right. \\
& \left.\times\left(p_{2} p_{3}\right)-O_{00 L}^{\prime \prime} m_{\chi}^{2}\left(p_{2} p_{4}\right)\right]
\end{aligned}
$$

In the limit $m_{\chi} \gg E_{\nu}$, we have

$$
s \simeq m_{\chi}^{2}, \quad t \simeq-2\left(p_{2} p_{4}\right), \quad u \simeq m_{\chi}^{2},
$$

and

$$
\begin{aligned}
& \left(p_{1} p_{2}\right) \simeq\left(p_{2} p_{3}\right) \simeq m_{\chi} E_{\nu}, \\
& \left(p_{1} p_{4}\right) \simeq\left(p_{3} p_{4}\right) \simeq m_{\chi} E_{\nu}, \\
& \left(p_{2} p_{4}\right) \simeq E_{\nu}^{2}(1-\cos \theta) .
\end{aligned}
$$

We thus see that for $m_{\chi} \gg E_{\nu}$, the square of the matrix element, and thus the cross section, are proportional to $\left(E_{\nu} / m_{\chi}\right)^{2}$, and so they are enormously suppressed as the neutrinos cool. The cross section is thus, schematically,

$$
\sigma_{\mathrm{el}} \sim(\text { couplings })^{2}\left(E_{\nu}^{2} / m_{\chi}^{4}\right)
$$

and should be at most (for couplings of order unity) $10^{-46} \mathrm{~cm}^{2}$. A survey of the supersymmetric parameter space shows that when realistic couplings are included, the typical cross section is more like $10^{-53} \mathrm{~cm}^{2}$. The cross section can be enhanced if the sneutrino mass is nearly degenerate with the neutralino mass, in which case there can be an $s$ - or $u$-channel resonance. However, the sneutrino would have to be extraordinarily close in mass to the neutralino, and there is no reason why this should be so in supersymmetric models.

\section{KINETIC DECOUPLING MORE GENERALLY}

More generically, the annihilation cross section should be

$$
\sigma_{\text {ann }} \sim(\text { couplings })^{2} / m_{\chi}^{2},
$$

and this must be $\sim 10^{-38} \mathrm{~cm}^{2}$ if the neutralino is to be the dark matter. This refers to the cross section for annihilation to light quarks, leptons, and neutrinos. The elastic-scattering cross section will be related to this by crossing symmetry, but with the correct kinematic factor, we obtain an elastic cross section like Eq. (17) (but with $E_{\nu}$ replaced more generally by the light-fermion energy). The rate at which a given neutralino will scatter from these light particles will be $\Gamma_{\mathrm{el}}$ $\sim n_{f} \sigma_{\mathrm{el}}$, where $n_{f} \sim T^{3}$ is the light-particle density, $T$ is the 
temperature, and at this temperature, the elastic-scattering cross section will be $\sigma_{\mathrm{el}} \sim 10^{-37} \mathrm{~cm}^{2}\left(T / m_{\chi}\right)^{2}$. Equating this elastic-scattering rate to the expansion rate, $H$ $\sim T^{2} / m_{\mathrm{Pl}}$, we find that neutralinos should undergo kinetic decoupling at

$$
T_{\text {kin dec }} \sim \operatorname{MeV}\left(m_{\chi} / \mathrm{GeV}\right)^{2 / 3} .
$$

Thus, after the neutralino freezes out at a temperature $T$ $\sim m_{\chi} / 20$, it will remain in kinetic equilibrium with the plasma and then undergo kinetic decoupling shortly thereafter. For neutralino masses $10 \mathrm{GeV} \lesssim m_{\chi} \lesssim 1000 \mathrm{GeV}$, this takes place well before neutrino decoupling and electronpositron annihilation.

At temperatures $T \lesssim 100 \mathrm{MeV}$, the baryons will be in the form of neutrons and protons, and shortly thereafter, $25 \%$ of the mass will be bound up in helium nuclei. The neutralinonucleon cross section required to maintain neutralino kinetic equilibrium at a temperature $T \sim \mathrm{MeV}$ is roughly $10^{-35} \mathrm{~cm}^{2}$. This cross section is extremely high for supersymmetric models (typical cross sections are $\lesssim 10^{-43} \mathrm{~cm}^{2}$ ), and is in fact ruled out by null searches for energetic neutrinos from weakly interacting massive particle (WIMP) annihilation in the Sun and Earth [16].

\section{CONCLUSIONS}

We have shown that the cross sections for elastic scattering of neutrinos and photons from neutralinos are far too small to keep neutralinos in kinetic equilibrium until $T$ $\sim 10-100 \mathrm{eV}$, when the Hubble length encloses dwarf- galaxy masses. Moreover, the cross sections required for collisional damping may be ten orders of magnitude bigger, in which case neutrino and photon elastic scattering are that much further from being able to provide collisional damping on these scales. Therefore, elastic scattering of neutralinos from neutrinos or photons cannot erase structure on subgalactic scales.

We have moreover argued that neutralinos should generically decouple kinetically from the primordial plasma shortly after they cease annihilating, above temperatures $T \sim \mathrm{MeV}$ (at which time a Hubble volume encloses no more than a solar mass of baryons). Neutralinos will thereafter act as collisionless cold dark matter and thus be incapable of altering the power spectrum on scales relevant for galaxy and largescale-structure formation.

Note added in proof. Shortly after the publication of our paper, another paper [17] which also discussed kinetic decoupling of cold dark matter came out.

\section{ACKNOWLEDGMENTS}

X.C. acknowledges the hospitality of the TAPIR group at Caltech where part of this work was completed. X.C. was supported in part by the U.S. Department of Energy under grant No. DE-FG02-91ER40690. M.K. was supported by the DOE under DE-FG03-92-ER40701, the NSF under AST0096023, and NASA under NAG5-8506. X.Z. was supported in part by the National Natural Science Foundation of China and the Ministry of Science and Technology of China under grant No. NKBRSF G19990754.
[1] G. Jungman, M. Kamionkowski, and K. Griest, Phys. Rep. 267, 195 (1996).

[2] L. Bergstrom, Rep. Prog. Phys. 63, 793 (2000).

[3] C. Boehm, P. Fayet, and R. Schaeffer, astro-ph/0012504.

[4] B. Moore et al., Astrophys. J. Lett. 524, L19 (1999).

[5] A. Klypin et al., Astrophys. J. 522, 82 (1999).

[6] J. E. Gunn et al., Astrophys. J. 223, 1015 (1978).

[7] C. Schmid, D. J. Schwarz, and P. Widerin, Phys. Rev. D 59, 043517 (1999)

[8] See, e.g., E. W. Kolb and M. S. Turner, The Early Universe (Addison-Wesley, Redwood City, 1990); P. J. E. Peebles, Principles of Physical Cosmology (Princeton University Press, Princeton, 1993).
[9] S. Rudaz, Phys. Rev. D 39, 3549 (1989).

[10] L. Bergstrom, Phys. Lett. B 225, 372 (1989).

[11] G. F. Giudice and K. Griest, Phys. Rev. D 40, 2549 (1989).

[12] G. Jungman and M. Kamionkowski, Phys. Rev. D 51, 3121 (1995).

[13] L. Bergstrom and P. Ullio, Nucl. Phys. B504, 27 (1997).

[14] Z. Bern, P. Gondolo, and M. Perelstein, Phys. Lett. B 411, 86 (1997).

[15] H. E. Haber and G. L. Kane, Phys. Rep. 117, 75 (1985).

[16] See, e.g., M. Kamionkowski, K. Griest, G. Jungman, and B. Sadoulet, Phys. Rev. Lett. 74, 5174 (1995); P. Ullio, M. Kamionkowski, and P. Vogel, hep-ph/0010036.

[17] S. Hoffman, D. J. Schwarz, and H. Stöcker, astro-ph/0104173. 